\title{
Abiraterone acetate withdrawal syndrome: Speculations on the underlying mechanisms
}

\author{
TOMONORI KATO ${ }^{1,2}$, AKIRA KOMIYA ${ }^{3}$, JOJI YUASA ${ }^{1}$, KANYA KAGA $^{1}$, \\ MAYUKO KAGA $^{1}$, SATOKO KOJIMA ${ }^{2}$, YUKIO NAYA ${ }^{2}$ and SHIGEO ISAKA ${ }^{1}$ \\ ${ }^{1}$ Division of Urology, Kuki General Hospital (Formerly Satte General Hospital), Saitama 346-8530; \\ ${ }^{2}$ Department of Urology, Teikyo University Chiba Medical Center, Chiba 299-0111; \\ ${ }^{3}$ Department of Urology, Chiba University Graduate School of Medicine, Chiba 260-8670, Japan
}

Received September 11, 2017; Accepted November 21, 2017

DOI: $10.3892 / \mathrm{ol} .2017 .7628$

\begin{abstract}
A 72-year-old man initially presented with lumbar and right chest pain, but was later found out to also have an elevated prostate-specific antigen (PSA) level at 2,000.0 ng/ml. Further evaluation disclosed metastatic prostate cancer involving the bones and lymph nodes. The patient was initially treated with combined androgen blockade (CAB) with leuprolide acetate and bicalutamide. After 6 months of CAB, the patient's PSA level began to rise from the nadir $(85.1 \mathrm{ng} / \mathrm{ml})$ to $113.3 \mathrm{ng} / \mathrm{ml}$. Bicalutamide was withdrawn in anticipation of anti-androgen withdrawal syndrome and the PSA level declined temporally. However, it increased up to $517.0 \mathrm{ng} / \mathrm{ml}$ thereafter. Consequently, a year after $\mathrm{CAB}$, abiraterone acetate (AA) was initiated at a standard dose of $1,000 \mathrm{mg}$ daily in combination with $10 \mathrm{mg}$ of prednisolone. PSA rapidly decreased to the nadir of $20.1 \mathrm{ng} / \mathrm{ml}$ thereafter. The PSA level remained stable until 2 years after AA administration. However, he decided to reduce the dose of AA to half of the standard dose (500 mg daily). Contrary to our expectations, the serum PSA level promptly decreased to a nadir of $8.1 \mathrm{ng} / \mathrm{ml}$. Thereafter, the PSA level remained stable until 3 years and 9 months after AA administration. Subsequently,
\end{abstract}

Correspondence to: Dr Tomonori Kato, Department of Urology, Teikyo University Chiba Medical Center, 3426-3 Anesaki, Ichihara-shi, Chiba 299-0111, Japan

E-mail:.jpn3899kato@yahoo.co.jp

Abbreviations: AA, abiraterone acetate; D4A, D-4-abiraterone; AAWS, abiraterone acetate withdrawal syndrome; AWS, anti-androgen withdrawal syndrome; PSA, prostate-specific antigen; ACTH, adrenocorticotropic hormone; mCRPC, metastatic castration-resistant prostate cancer; $\mathrm{CAB}$, combined androgen blockade; mAR, mutant androgen receptor; mARA, mutant androgen receptor activation; LBD, ligand-binding domain; MRI, magnetic resonance imaging; $\mathrm{CT}$, computed tomography

Key words: metastatic castration refractory prostate cancer, abiraterone acetate, prednisolone, progesterone, anti-androgen withdrawal syndrome, abiraterone acetate withdrawal syndrome, mutant androgen receptor activation, alternative ligand the patient stopped taking AA and prednisolone. However, to our surprise, the patient's serum PSA level decreased further to $<1.0 \mathrm{ng} / \mathrm{ml}$ after AA discontinuation. His PSA remained $<1.0 \mathrm{ng} / \mathrm{ml}$ without clinical or radiological progression for 1 year after AA withdrawal. Recently, it was reported that cessation of AA is associated with AA withdrawal syndrome in metastatic castration-resistant prostate cancer, defined as a PSA decrease after AA discontinuation, mimicking anti-androgen withdrawal syndrome. In the present study, explanations of the mechanisms underlying this phenomenon were explored, including mutant AR activation by alternative ligands.

\section{Introduction}

Recently, it was reported that cessation of abiraterone acetate (AA) is associated with an AA withdrawal syndrome (AAWS) in metastatic castration-resistant prostate cancer (mCRPC) which was first reported in 2012 (1). AAWS is characterized by a transient prostate-specific antigen (PSA) decrease after AA discontinuation, mimicking anti-androgen withdrawal syndrome (AWS) (2). The use of AA in patients with mCRPC has been increasing since its registration. With the widespread use of AA, AAWS has become gradually recognized. So far, limited evidence is available on this phenomenon and the mechanisms leading to AAWS is still difficult to explain. In this manuscript, we report the case of a patient who had a drastic PSA decrease after stopping AA. We also provide a review of recent literature and describe potential explanation of the mechanism underlying this phenomenon. Particularly, activation of mutant androgen receptor (mAR) by probable alternative ligands such as prednisolone, progesterone, AA itself and its steroidal metabolites are mainly discussed in this manuscript.

Written informed consent was obtained from the patient for publication of this case report and any accompanying images. No ethics approval sought as this was a case report with no direct impact on patient outcome.

\section{Case report}

A 72-year-old man complaining of lumbar and right chest pain was referred to our hospital in June 2011 for further evaluation of serum PSA elevation that had reached 2,000.0 ng/ml. 
Prostate needle biopsy was performed and pathological examination showed prostatic adenocarcinoma and the Gleason score of 4+4. Magnetic resonance imaging (MRI) showed massive prostate tumor that invaded the rectum and the left seminal vesicle directly (Fig. 1A). Computed tomography (CT) revealed metastasis of the pelvic lymph nodes and bone scintigraphy revealed multiple bone metastasis (Fig. $1 \mathrm{~B}$ and $\mathrm{C}$ ). He was diagnosed with prostate cancer, cT4N1M1b.

The PSA kinetics during treatment are shown in Fig. 2. Initially, combined androgen blockade (CAB) with leuprolide acetate and bicalutamide had been started. The serum PSA level had decreased to $85.1 \mathrm{ng} / \mathrm{ml}$ in November 2011, but then gradually increased again to $113.3 \mathrm{ng} / \mathrm{ml}$ in February 2012. Then, bicalutamide was discontinued to determine AWS.

Subsequently the serum PSA level had temporarily decreased however, then gradually increased again and serum PSA increased to $517.0 \mathrm{ng} / \mathrm{ml}$ in July 2012. Consequently, AA was initiated at the standard dose of $1,000 \mathrm{mg}$ daily in combination with prednisolone (10 mg daily). PSA rapidly decreased to a nadir of $20.1 \mathrm{ng} / \mathrm{ml}$, thereafter, his PSA had remained stable between $20-50 \mathrm{ng} / \mathrm{ml}$ during this period.

In July 2014, he confessed that he had felt nauseous after taking AA for a long time. Thereafter, arbitrarily on his own, he reduced taking AA to half of the standard dose (500 mg daily). However, contrary to our expectations, his serum PSA level promptly decreased to a nadir of $8.1 \mathrm{ng} / \mathrm{ml}$. At that time, he still continued taking prednisolone at the standard dose of $10 \mathrm{mg}$. Thereafter, his PSA has become a lot lower between $8-16 \mathrm{ng} / \mathrm{ml}$ than before. Although the association between AA and his nausea is unclear, his nausea resolved spontaneously thereafter.

Subsequently, in April 2016, he complained of steroid purpura on bilateral forearms, which may not require particular treatment. We recommended him to continue taking AA. However, he self-indulgently stopped taking AA. He had also quit taking prednisolone at the same time. Surprisingly, his serum PSA levels decreased to $<1.0 \mathrm{ng} / \mathrm{ml}$ after AA discontinuation without radiological progression. The patient is still well and alive 1 year after AA cessation without a biological, clinical or radiological progression under leuprolide acetate (Fig. 3A and B).

\section{Discussion}

Similar to AWS, a PSA decrease after AA discontinuation, namely AAWS, is recently recognized and reported in mCRPC (2). Initially, AAWS had been thought to be minor short-term event (3). AAWS with a biochemical response $>50 \%$ were retrospectively observed in $6 \%$ of the patients who stopped AA because of disease progression (3). However, in the subsequent manuscript, AAWS has proved to be not so uncommon event. PSA response $>50 \%$ was retrospectively observed in $15 \%$ ( 3 of 26) of mCRPC patients after stopping AA (4). It was also demonstrated that AAWS was observed in $16 \%$ (3 of 19) mCRPC patients and 2 of them associated with clinical and radiological response (4). It has gradually become known that AAWS can occasionally induce a long-lasting biochemical response accompanied by a clinical and radiological improvement (4). More recently, it was also suggested that patients who experienced AAWS may have better survival outcomes (5).
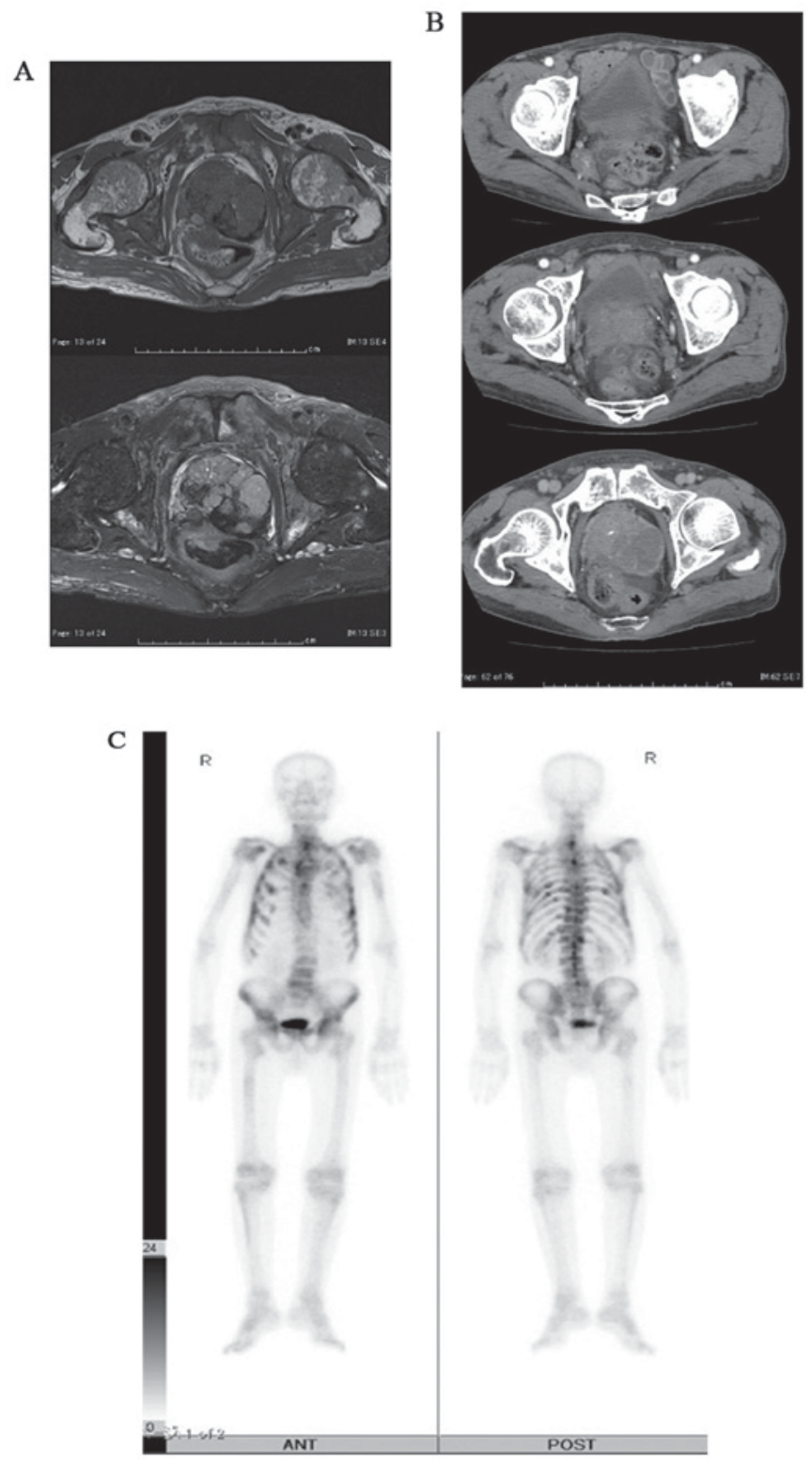

Figure 1. The patient was diagnosed with prostate cancer T4N1M1b. (A) Magnetic resonance imaging showed massive prostate tumor that invaded the rectum and the left seminal vesicle directly. (B) Computed tomography revealed metastasis of the pelvic lymph nodes metastasis. (C) Bone scintigraphy showed multiple bone metastasis.

Currently proposed leading mechanisms of resistance to AA includes activation of mutant AR by alternative ligands, ligandless AR activation by constitutively active AR variants lacking the ligand-binding domain and/or cross-talk with relative signaling pathways (6). Among these mechanisms, AR mutations in the hormone-binding domain which allow activation by alternative ligands other than testosterone is supposed to be the main cause of AAWS.

Among withdrawal syndromes, AWS associated with anti-androgens has been mainly investigated. AWS is the phenomenon characterized by tumor regression and decline in serum PSA levels on discontinuation of the anti-androgen (7). AR mutations in the ligand-binding domain (LBD) and/or hinge region, such as T877A or H874Y, are speculated to be responsible for stimulating effects of anti-androgens on prostate cancer (8). Recently, F876 L AR 


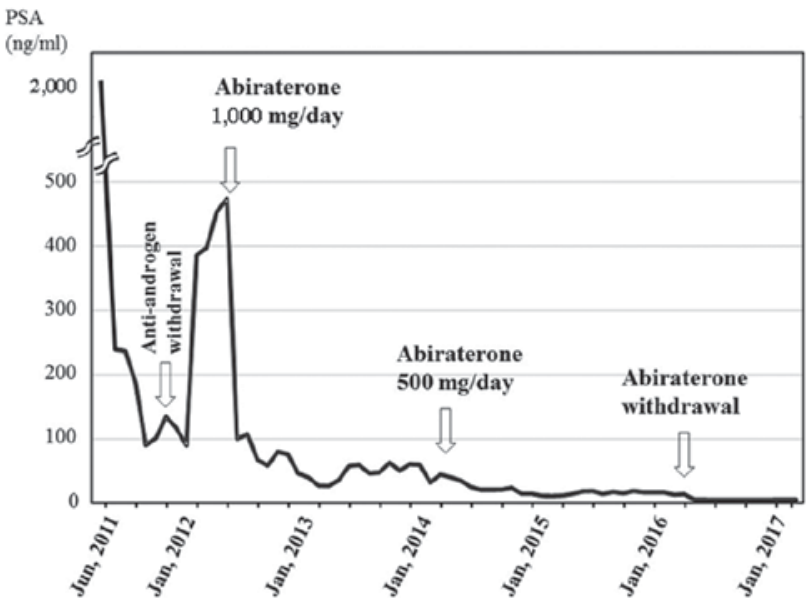

Figure 2. The graph illustrates time-course changes of serum prostate-specific antigen (PSA) levels and therapeutic agents. Initially he reduced taking abiraterone acetate (AA) to half of the standard dose (500 $\mathrm{mg}$ daily). However, his serum PSA level promptly decreased to a nadir of $8 \mathrm{ng} / \mathrm{ml}$. Subsequently, he stopped taking AA and at the same time quit taking prednisolone. However, his serum PSA level further decreased to $<1.0 \mathrm{ng} / \mathrm{ml}$, after AA discontinuation.

mutation, which converted enzalutamide into an AR agonist, is also reported (6). Various $\mathrm{mARs}$ can reduce ligand binding specificity, and induce mAR activation (mARA) by several ligands other than testosterone. These alternative ligands are not restricted only to anti-androgen. Various mARs are demonstrated to be activated by various alternative ligands, such as progesterone, estradiol, adrenal androgens, hydrocortisone or anti-androgens (6). Several withdrawal syndromes associated with hormonal agents other than anti-androgens are also reported (6).

With regard to AAWS, the following ligands has been supposed to bind to LBD of $\mathrm{mAR}$ : A) prednisolone taken with AA, B) slightly elevated progesterone induced by AA administration and C) AA itself or steroidal metabolites of AA.

AA is generally administered in association with prednisolone, at a daily dose of $10 \mathrm{mg}$, to prevent the mineralocorticoid excess caused by the loss of a negative feedback of the adrenocorticotropic hormone (ACTH) (9). In most cases, both AA and prednisolone were stopped at the same time. Therefore, it had been hypothesized that prednisolone taken with AA is responsible for the withdrawal response (4). Prednisolone is also known to act as alternative ligand for certain mAR (6). It is probable that AAWS might partly depend on prednisolone discontinuation rather than AA discontinuation. However, it is difficult to explain this phenomenon only by means of prednisolone. As a matter of fact, AAWS is documented even in those who continued steroids after AA cessation (10). The underlying mechanisms other than prednisolone are speculated from our case too. Our case initially reduced AA to half dose with continuing prednisolone at the standard dose of $10 \mathrm{mg}$, which results in more decreased PSA level.

AA is a potent CYP17 inhibitor that blocks androgen synthesis which drastically reduces circulating and tissue androgen levels which otherwise increases steroids upstream of CYP17 including progesterone. Serum and tissue progesterone level slightly increases through AA
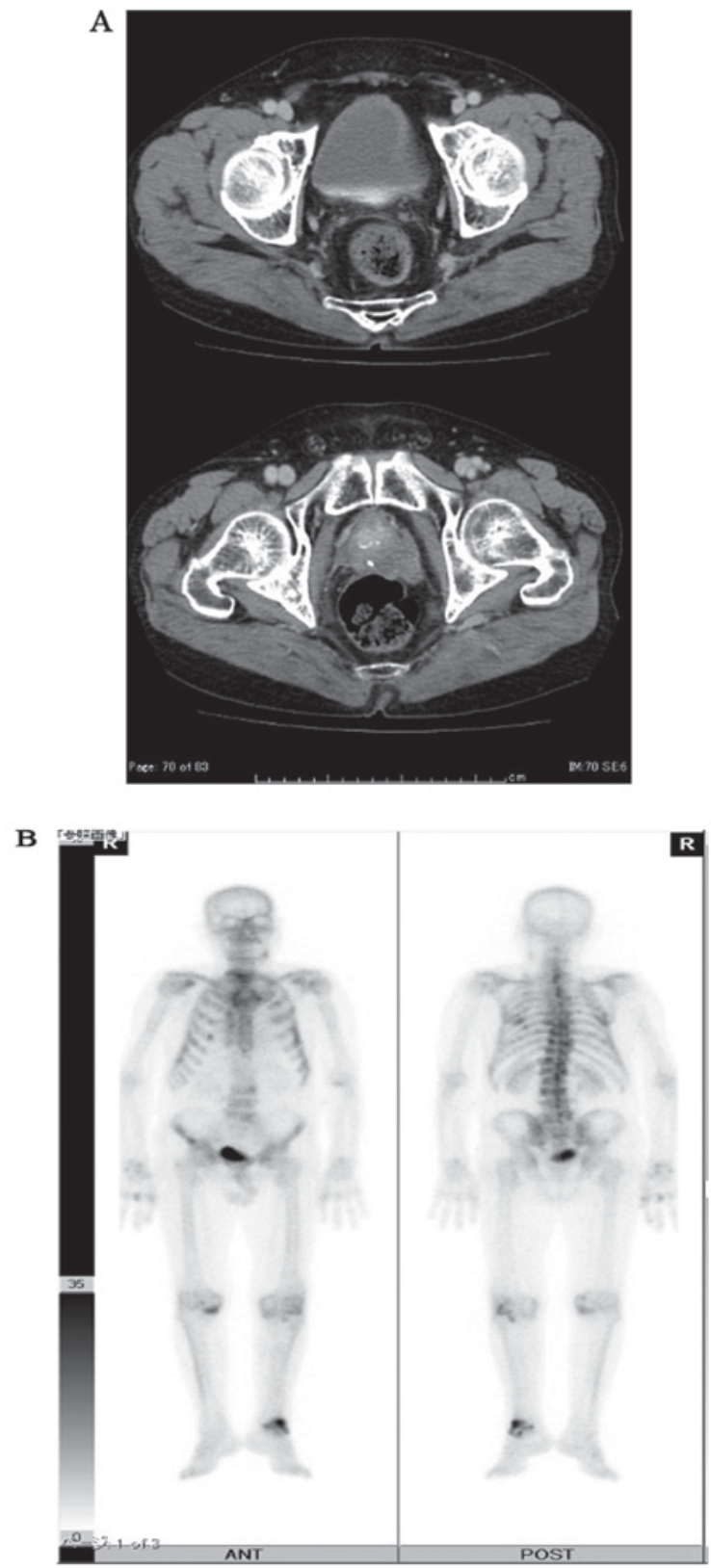

Figure 3. (A) Computed tomography and (B) bone scintigraphy have shown no evidence of disease progression since the abiraterone acetate withdrawal.

administration (11). Progesterone can also activate several mARs (11). Among them, T878A mAR is suggested to be upregulated through AA treatment, which is one of the possible mechanisms of AAWS (12). Due to the agonistic effect on mAR, even a small amount of progesterone might be responsible for AAWS.

Participation of AA itself and/or steroidal metabolites of $\mathrm{AA}$ are also suggested as possible mechanism for AAWS. AA itself could have direct AR antagonistic properties and AAWS might possibly be linked to direct abiraterone-AR interactions (13). Moreover, multiple steroidal metabolites of AA such as D-4-abiraterone (D4A) and 5a-abiraterone which are previously unappreciated, has recently been identified. AA is metabolized to D4A, which act as an AR antagonist (14). D4A is converted to 5a-abiraterone, which act as an AR agonist (15). Furthermore, various steroidal metabolites of AA had been 
successively detected and these metabolites are speculated to interact directly with wildtype or mutant AR (16).

Conventionally, AWS is defined as a disease response and clinical improvement after discontinuation of the anti-androgen in patients developing progressive prostate cancer during CAB (7), however, we emphasize that the definition of withdrawal syndrome should be reconsidered. As mentioned above, withdrawal syndromes could be derived by various ligands other than anti-androgen including progesterone, estradiol, corticosteroids, AA and other steroidal derivatives. Taking account of these phenomenon, these should all be widely categorized an 'alternative-ligand withdrawal syndromes'. Besides, withdrawal syndromes are not the phenomenon restricted to disease progression periods. In our patient, AAWS with additional PSA decreases were observed when his PSA levels once reached his PSA nadir. From another point of view, his nadir PSA had never reached an undetectable level during AA treatment which indicated that drug resistance for AA potentially existed. Recently, there have been several reports regarding the relationships between nadir PSA level and clinical outcome. In the population-based database includes 26,272 Japanese men with prostate cancer, it is demonstrated that patients with nadir PSA levels $<0.2 \mathrm{ng} / \mathrm{ml}$ following primary androgen deprivation therapy had better clinical outcomes than patients with nadir PSA levels $\geq 0.2 \mathrm{ng} / \mathrm{ml}$ which indicates underlying drug resistance (17). Even while on novel AR targeting therapies, drug resistance may concealed if PSA did not reach undetectable levels.

Although the mechanisms underlying AAWS requires further study, clinicians should pay attention to this phenomenon. Considering the frequency of AAWS, it might not be proper to routinely anticipate AAWS. However, when patient's conditions allow, it would be acceptable to wait for a brief period before starting further therapy after AA progression because it would be a good opportunity to presume existing drug resistance including mARA. Through evaluation of PSA kinetics on daily medical examination, useful information on managing mCRPC can sometimes be obtained.

\section{References}

1. Gauthier H, Bousquet G, Pouessel D and Culine S: Abiraterone acetate withdrawal syndrome: Does it exist? Case Rep Oncol 5: 385-387, 2012.

2. Witjes JA: A case of abiraterone acetate withdrawal. Eur Urol 64: 517-518, 2013.

3. Albiges L, Auclin E, Rousseau B, Boughalem E, Levy A, Loriot Y, Palma MD, Massard C and Fizazi K: Is there a withdrawal syndrome with abiraterone acetate (AA)? J Clin Oncol 31: 89, 2013.
4. Caffo O, Palermo A, Veccia A, Maines F, Chierichetti F, Berruti A and Galligioni E: Biochemical and objective response to abiraterone acetate withdrawal: Incidence and clinical relevance of a new scenario for castration-resistant prostate cancer. Urology 82: 1090-1093, 2013.

5. Caffo O, Maines F, Trentin C, Veccia A and Galligioni E: Long-term outcomes and predictive factors in patients (pts) with metastatic castration-resistant prostate cancer (mCRPC) showing abiraterone withdrawal syndrome (AWS) after docetaxel (DOC) treatment. J Clin Oncol 34 (2 Suppl): S324, 2016.

6. Lorente D, Mateo J, Zafeiriou Z, Smith AD, Sandhu S, Ferraldeschi R and de Bono JS: Switching and withdrawing hormonal agents for castration-resistant prostate cancer. Nat Rev Urol 12: 37-47, 2015

7. Paul R and Breul J: Anti-androgen withdrawal syndrome associated with prostate cancer therapies: Incidence and clinical significance. Drug Saf 23: 381-390, 2000.

8. Taplin ME, Bubley GJ, Shuster TD, Frantz ME, Spooner AE, Ogata GK, Keer HN and Balk SP: Mutation of the androgen-receptor gene in metastatic androgen-independent prostate cancer. N Engl J Med 332: 1393-1398, 1995.

9. de Bono JS, Logothetis CJ, Molina A, Fizazi K, North S, Chu L, Chi KN, Jones RJ, Goodman OB Jr, Saad F, et al: Abiraterone and increased survival in metastatic prostate cancer. N Engl J Med 364: 1995-2005, 2011.

10. Azad AA and Eigl BJ: Evaluation of prostate-specific antigen response following cessation of abiraterone acetate: Is there evidence for a withdrawal syndrome? Eur Urol 65: 504-505, 2014.

11. Taplin ME, Montgomery B, Logothetis CJ, Bubley GJ, Richie JP, Dalkin BL, Sanda MG, Davis JW, Loda M, True LD, et al: Intense androgen-deprivation therapy with abiraterone acetate plus leuprolide acetate in patients with localized high-risk prostate cancer: Results of a randomized phase II neoadjuvant study. J Clin Oncol 32: 3705-3715, 2014.

12. Chen EJ, Sowalsky AG, Gao S, Cai C, Voznesensky O, Schaefer R, Loda M, True LD, Ye H, Troncoso P, et al: Abiraterone treatment in castration-resistant prostate cancer selects for progesterone responsive mutant androgen receptors. Clin Cancer Res 21: 1273-1280, 2015

13. Richards J, Lim AC, Hay CW, Taylor AE, Wingate A, Nowakowska K, Pezaro C, Carreira S, Goodall J, Arlt W, et al: Interactions of abiraterone, eplerenone, and prednisolone with wild-type and mutant androgen receptor: A rationale for increasing abiraterone exposure or combining with MDV3100. Cancer Res 72: 2176-2182, 2012.

14. Li Z, Bishop AC, Alyamani M, Garcia JA, Dreicer R, Bunch D, Liu J, Upadhyay SK, Auchus RJ and Sharifi N: Conversion of abiraterone to D4A drives anti-tumour activity in prostate cancer. Nature 523: 347-351, 2015.

15. Li Z, Alyamani M, Li J, Rogacki K, Abazeed M, Upadhyay SK, Balk SP, Taplin ME, Auchus RJ and Sharifi N: Redirecting abiraterone metabolism to fine-tune prostate cancer anti-androgen therapy. Nature 533: 547-551, 2016.

16. Caffo O and Sharifi N: Could steroidal abiraterone metabolites possibly explain abiraterone withdrawal syndrome? Eur Urol 70: 898-899, 2016.

17. Kitagawa $Y$, Ueno S, Izumi K, Mizokami A, Hinotsu S, Akaza $H$ and Namiki M: Nadir prostate-specific antigen (PSA) level and tome to PSA nadir following primary androgen deprivation therapy as independent prognostic factors in a Japanese large-scale prospective cohort study (J-CaP). J Cancer Res Clin Oncol 140: 673-679, 2014. 